\title{
STRONGLY REVERSIBLE MANIFOLDS
}

\section{J. HOCKING and P. DOYLE}

(Received 9 May 1980)

Communicated by G. Virsik

\begin{abstract}
The results here concern bijective continuous functions from one connected separable $n$-manifold $M$ to another $N$. If $M$ has the property that every such function is necessarily a homeomorphism, then $M$ is said to be strongly reversible. Strongly reversible manifolds having only compact boundary components are completely characterized.
\end{abstract}

1980 Mathematics subject classification (Amer. Math. Soc.): 57 N 15, 57 A 05.

This is a sequel to the work on reversible manifolds by Doyle and Hocking (1976). There a connected separable manifold is defined to be reversible if every continuous self-bijection is necessarily a homeomorphism. (More general reversible spaces have been studied by Pettey (1970) and by Rajagopalan and Wilansky (1966).)

A connected separable $n$-manifold $M$ is strongly reversible if every continuous bijection of $M$ to an $n$-manifold is necessarily a homeomorphism. (To give an easy example note that $M=[0,1) \times(0,1)$ is reversible but not strongly reversible.)

Bijective images of manifolds having non-compact boundary components can present unusual complications. The first theorem below and the example which follows it point to such problems. They also explain our limiting consideration, in this paper, to the case of manifolds having only compact boundary components.

ThEOREM 1. Let $M$ be an n-manifold having a boundary component $C$ that embeds in $E^{n-1}$. Then $M$ is not strongly reversible.

Proof. $C$ is collared in $M$ and so has a closed neighborhood homeomorphic to $C \times[0,1]$ in $M$. We shall abuse the language and call the neighborhood $C \times[0,1]$,

(c) Copyright Australian Mathematical Society 1983 
also. Since $C$ is not compact, there is locally flat ray $l$ in $C \times 1 / 2$ such that $l$ is closed in $C \times 1 / 2$. Swell this ray into a set $K$ in $C \times(0,1)$, where $K$ is a closed $n$-cell with a boundary point removed and where $\operatorname{Bd} K$ (boundary of $K$ ) is bicollared. If $J$ is any open set containing $K$ there is a homeomorphism $h$ of $M-K$ onto $M$ with $h$ fixed outside of $U$. We now see that there is a pasting of $C$ onto a portion of the end of $M-K$ at the missing $\mathrm{Bd} K$. Thus $M$ is not strongly reversible.

Note that it may be difficult to generalize the construction just given. Suppose $F$ is a fake 3 -sphere and $X$ is $F$ with a point removed. Then $X$ fails to embed in $E^{3}$ but $x \times[0,1)$ is not strongly reversible.

The following example exhibits another perhaps unexpected property of bijective maps on manifolds. Let $M_{1}$ be the planar manifold consisting of the sets

$$
\{(x, y) \mid 0 \leqslant x<+\infty,-1<y \leqslant 0\}-\{(n, 0) \mid n=0,1,2, \ldots\}
$$

and

$$
\bigcup_{n=1}^{\infty}\left\{(x, y) \mid n-1 \leqslant x<n-\sqrt{y-y^{2}}, 0<y<1\right\} .
$$

(Of course, $M_{1}$ is merely an open 2-cell with infinitely many open boundary segments added. It is presented like this to make the next step easy.) On $M_{1}$ define the map

$$
\begin{aligned}
f_{1}(x, y) & =(x, y) & & \text { if } y \leqslant 0 \\
& =(g(x, y), y) & & \text { if } 0<y<1
\end{aligned}
$$

where

$$
g(x, y)=[x]+\frac{x-[x]}{1-\sqrt{y-y^{2}}},
$$

$[x]$ denoting the greatest integer function. The function $f_{1}$ stretches the half-open segment $\left[n-1, n-\sqrt{y-y^{2}} \times\{y\}\right)$ to $[n-1, n)$. We see that $f_{1}$ is continuous and bijective while $f_{1}^{-1}$ fails to be continuous. Also note that $f_{1}\left(M_{1}\right)=M_{2}^{\prime}$ consists of the set

$$
\{(0, y) \mid 0<y<1\} \cup\{(x, y) \mid 0<x<+\infty,-1<y<1\}
$$

with the integer points $(n, 0)$ removed.

Next define the map $f_{2}(x, y)=(h(x), y)$ where $h$ is a homeomorphism of $[0,+\infty)$ onto $[0,2 \pi)$ followed by the exponential map of $[0,2 \pi)$ onto $S^{1}$. Note that $f_{2}$ carries $M_{2}^{\prime}$ onto the cylinder $S^{i} \times(-1,1)$ with a convergent sequence of points, and the limit point of this sequence, removed. If this latter manifold is denoted by $M_{2}$, then $f=f_{2} \circ f_{1}: M_{1} \rightarrow M_{2}$ is a bijection and $f \mid \mathrm{Bd} M_{1}$ is not a homeomorphism. It is precisely this situation we can avoid in the setting of this 
study, namely, restricting attention to manifolds having only compact boundary components.

THEOREM 2. Let $f: M_{1} \rightarrow M_{2}$ be a bijective map of connected n-manifolds where each component of $\mathrm{Bd} M_{1}$ is compact and $\mathrm{Bd} M_{2}=\varnothing$. Then $f \mid \mathrm{Bd} M_{1}$ is an embedding.

Proof. First note that $f \mid B$ is a homeomorphism for each component $B$ of Bd $M_{1}$. If $f \mid \mathrm{Bd} M_{1}$ is not an embedding there must be a sequence $\left\{B_{i}\right\}$ of components of $\mathrm{Bd} M_{1}$ and points $y_{i} \in f\left(B_{i}\right)$ such that $\left\{y_{i}\right\} \rightarrow y_{1}$.

Note that $f\left(B_{1}\right)$ has an open connected neighborhood $A$ in $M_{2}$ having compact closure and that $A$ can be chosen so that $f\left(B_{1}\right)$ separates $A$. Also $f\left(B_{1}\right)$ is an ANR so $A$ may be selected such that $H_{n-1}(A, Z)$ is finitely generated. (See page 81 , Corollary 8.7 of Dold (1972).) None of the images $f\left(B_{i}\right)$ separate $M_{2}$ so at most finitely many can lie in $A$. It follows that we may further select $A$ so as to contain $f\left(B_{1}\right)$ and no other images $f\left(B_{i}\right)$. Hence we have $f\left(B_{i}\right) \cap A \neq \varnothing$ and $f\left(B_{i}\right) \cap$ $\left(M_{2}-A\right) \neq \varnothing$ for all $i$.

Let $Q_{j}$ be a closed $n$-cell neighborhood of $y_{1}$ of diameter $<1 / j$ for each positive $j$. Clearly, each set

$$
Q_{j} \cup f\left(B_{1}\right) \cup\left(\bigcup_{i=z}^{\infty}\left(f\left(B_{i}\right) \cap A\right)\right)
$$

contains connected sets which, when closed, contain both $y_{1}$ and points of $\bar{A}-A$. Let $C_{j}$ be the maximal such continuum for each $j$ and set

$$
L=\bigcap_{j=2}^{\infty} C_{j} .
$$

Surely $L$ is a continuum joining $y_{1}$ to $\bar{A}-A$ while $L$ lies in $f\left(\mathrm{Bd} M_{1}\right)$. But now $L \cap f\left(\mathrm{Bd} M_{1}\right)$ presents $L$ as a countable union of disjoint compact sets. Theorem 6 on page 173 of Kuratowski (1968) shows this is impossible and completes the proof.

The most general case treated here is that of a bijective map $f$ from a connected $n$-manifold $M_{1}$ having only compact boundary components to an $n$-manifold $M_{2}$. Since $f^{-1}\left(\mathrm{Bd} M_{2}\right)$ must lie in Bd $M_{1}$, we may replace $f$ with a restriction from $M_{1}-f^{-1}\left(\mathrm{Bd} M_{2}\right)$ to Int $M_{2}$ (interior of $\left.M_{2}\right)$. Thus the assumption $\mathrm{Bd} M_{2}=\varnothing$ is no limitation. If, in this context, $f$ is not a homeomorphism, then from the proof of Theorem 3 in Doyle and Hocking (1976) there exists a component $C$ of $\mathrm{Bd} M_{1}$ such that $f(C)$ lies in Int $M_{2}$. By Theorem 2 above $f(C)$ has a neighborhood $A$ in $M_{2}$ that meets no other component of $f\left(\operatorname{Bd} M_{1}\right)$. Because $f(C)$ separates $A$ but not 
$M_{2}, A$ can be selected to be the disjoint union of a collar on $f(C)$ and the image under $f$ of some end $\varepsilon$ of $M_{1}$. (Although the end $\varepsilon$ "fits" on one side of $f(C)$ it need not have a product, or even a fiber, structure. The bad complement of the Alexander horned sphere in $E^{3}$ is an example.) Clearly, $\varepsilon$ can be compactified with boundary $C$ in a sense more general than that of Eichorn (1978) and others. We say that an end $\varepsilon$ of an $n$-manifold $M$ is $C$-like if it can be compactified with the closed ( $n-1)$-manifold $C$ in the sense just described. (Obviously a $C$-like end must be isolated.) With this definition the main theorem can be stated.

THEOREM 3. Let $f: M_{1} \rightarrow M_{2}$ be a continuous bijection of the connected $n$-manifold $M_{1}$ to the n-manifold $M_{2}$. Assume that $\mathrm{Bd} M_{2}$ has only compact components and that $\operatorname{Bd} M_{2}=\varnothing$. There is an $M_{2}$ for which $f$ fails to be homeomorphism if and only if, for some component $C$ of $\mathrm{Bd} M_{1}, M_{1}$ has a $C$-like end.

In view of Theorem 3 the setting of many results of Eichorn (1978) can be generalized to the topological case.

The next result is immediate.

THEOREM 4. An n-manifold $M$ with only compact boundary components is strongly reversible if and only if, for each component $C$ of $\mathrm{Bd} M, M$ has no $C$-like end.

Combining Theorems 1 and 4 gives an easy proof of the next result.

THEOREM 5. A 2-manifold $M$ with nonempty boundary is strongly reversible if and only if $\mathrm{Bd} M$ has only compact components and $M$ has no $S^{1}$-like ends.

THEOREM 6. Let $M_{1}$ be an n-manifold having only compact boundary components and $\mathrm{Bd} M_{1} \neq \varnothing$. If $M_{2}$ is an n-manifold with $H_{n-1}\left(M_{2}, Z_{2}\right)=0$, then the only bijective maps $f: M_{1} \rightarrow M_{2}$ are homeomorphisms.

\section{References}

A. Dold (1972) Lectures in algebraic topologv (Springer Verlag).

P. H. Doyle and J. G. Hocking (1976), 'Continuous bijections on manifolds', J. Austral. Math. Soc. 22. $257-263$

J. Eichorn (1978), 'Die Kompaktifizierung offener Mannigfaltigkeiten zu geschlossenen I', Math. Nachr. 85. 5-30.

K. Kuratowski (1968), Topolog, Vol. 2 (Academic Press). 
D. H. Pettey (1970), 'One-to-one mappings into a plane', Fund. Math . 67, 209-218.

M. Rajagopalan and A. Wilansky (1966), 'Reversible topological spaces', J. Austral. Math. Soc. 6, 129-138.

Department of Mathematics

Michigan State University

Wells Hall

East Lansing, Michigan 48824

U.S.A. 